\title{
Surgical outcomes and multidisciplinary management strategy of Cushing's disease: a single-center experience in China
}

\author{
*Keyi Zhang, MD,1 Ming Shen, MD, PhD, ${ }^{1}$ Nidan Qiao, MD, PhD, ${ }^{1}$ Zhengyuan Chen, MD,1 \\ Wenqiang He, MD, PhD, ${ }^{1}$ Zengyi Ma, MD, PhD, ${ }^{1}$ Xuefei Shou, MD, PhD, ${ }^{1}$ Shiqi Li, MD, ${ }^{1}$ \\ Yao Zhao, MD, PhD, ${ }^{1}$ Li Pan, MD, PhD, ${ }^{1,2}$ Dan Liu, MD, ${ }^{3}$ Min He, MD, PhD, ${ }^{3}$ Zhaoyun Zhang, MD, PhD, ${ }^{3}$ \\ Yiming Li, MD, PhD, ${ }^{3}$ Zhenwei Yao, MD, PhD, ${ }^{4}$ Hongying Ye, MD, ${ }^{3}$ and Yongfei Wang, MD, PhD ${ }^{1}$ \\ 1'Department of Neurosurgery, and ${ }^{2}$ CyberKnife Center, Shanghai Huashan Institute of Neurological Surgery, Huashan Hospital, \\ Shanghai Medical School, Fudan University; and Departments of ${ }^{3}$ Endocrinology and ${ }^{4}$ Radiology, Huashan Hospital, Shanghai \\ Medical School, Fudan University, Shanghai, China
}

OBJECTIVE The primary aim of this study was to investigate the value of multidisciplinary team (MDT) management in treating patients with Cushing's disease (CD). The secondary aim was to assess the concordance of bilateral inferior petrosal sinus sampling (BIPSS) lateralization with intraoperative observations.

METHODS The authors recruited 124 consecutive patients (128 procedures) who had undergone endoscopic endonasal resection of adrenocorticotropic hormone-secreting pituitary adenomas from May 2014 to April 2018 and assessed their clinical characteristics, surgical outcomes, and adjuvant therapies. The criteria for surgical remission were normalized serum and urinary cortisol levels, which could be suppressed by a low-dose dexamethasone suppression test at 3-months' follow-up without adjuvant treatment.

RESULTS The remission rates of the 113 patients with long-term follow-up ( $20.3 \pm 12.2$ months) were $83.2 \%$ after surgery alone and $91.2 \%$ after adjuvant therapy. The surgical remission rates of macroadenomas, MRI-visible microadenomas, and MRI-negative tumors were $66.7 \%$ (12/18), 89.3\% (67/75), and 75\% (15/20), respectively $(p=0.039)$. The surgical remission rates had a trend of improvement during the study period (87.5\% in $2017-2018$ vs $76.5 \%$ in 2014 , p $=0.517)$. Multivariate regression analysis showed that a history of previous pituitary surgery $(\mathrm{OR} 0.300,95 \% \mathrm{Cl} 0.100-$ 0.903; $p=0.032$ ) and MRI-visible microadenoma (OR 3.048, 95\% Cl 1.030-9.019; $p=0.044$ ) were independent factors influencing surgical remission. The recurrence rate was $3.2 \%$ after a mean of 18 months after surgery. The remission rate of postoperative MDT management in patients with persistent disease was higher than non-MDT management (66.7\% vs $0 \%, p=0.033$ ). In cases with preoperative BIPSS lateralization, $84.6 \%$ (44/52) were concordant with intraoperative findings.

CONCLUSIONS MRI-visible microadenoma and primary surgery were independent predictors of surgical remission in CD. The MDT management strategy helps to achieve a better overall outcome. BIPSS may help to lateralize the tumor in MRI-negative/equivocal microadenomas.

https://thejns.org/doi/abs/10.3171/2020.3.FOCUS2067

KEYWORDS Cushing's disease; ACTH-secreting pituitary adenoma; endoscopic endonasal surgery; remission; multidisciplinary team; petrosal sinus sampling

\footnotetext{
ABBREVIATIONS $\mathrm{ACTH}=$ adrenocorticotropic hormone; BIPSS = bilateral inferior petrosal sinus sampling; $\mathrm{CD}=$ Cushing's disease; $\mathrm{cMRI}=$ conventional gadoliniumenhanced MRI; CS = Cushing's syndrome; HDDST = high-dose dexamethasone suppression test; LDDST = low-dose dexamethasone suppression test; MDT = multidisciplinary team; SPACE = sampling perfection with application-optimized contrasts using different flip angle evolutions; spMRI = 3D-SPACE MRI; SRT = stereotactic radiotherapy; TSS = transsphenoidal surgery; UFC = urinary free cortisol.
}

SUBMITTED January 30, 2020. ACCEPTED March 5, 2020

INCLUDE WHEN CITING DOI: 10.3171/2020.3.FOCUS2067.

${ }^{*}$ K.Z. and M.S. contributed equally to this work. 
A DRENOCORTICOTROPIC hormone (ACTH)-secreting pituitary adenoma, also known as Cushing's disease (CD), is clinically manifested as Cushing's syndrome (CS), usually including weight gain, central fat deposition, cutaneous purple striae, skin thinning, muscle wasting, and fatigue. CD accounts for 70\%-80\% of all causes of primary CS, with an incidence of about 2-3 per million population per year. ${ }^{3,8,16,23,32}$ Comorbidities related to hypercortisolism include diabetes mellitus, hypertension, and cardiovascular disease. Untreated patients or those with persistent disease have a poor prognosis, with a 5-year survival rate of approximately 50\%,6,8 Conversely, the long-term survival rate of patients with biochemical remission after surgery showed no difference with the normal population (standardized mortality ratio $0.95,95 \%$ CI 0.58-1.55).?

The first-line treatment of CD is surgical removal of the tumor by transsphenoidal surgery (TSS). In recent decades, the application of endoscopes in the approach for treating pituitary adenomas has gained considerable popularity due to the advantages of the endoscope, including a panoramic surgical view, increased illumination, and a close-up observation. We adopted the endoscopic endonasal approach in treating patients with CD starting in 2014.

The diagnosis and management of $\mathrm{CD}$ require the participation of multidisciplinary specialists. In addition, ACTH-secreting pituitary adenomas are known to be small in size, and thus difficult to detect on MRI, which increases the surgical risks. However, few studies have stressed the value of multidisciplinary team (MDT) management, both preoperatively and postoperatively, for CD. We hypothesize that: 1) close cooperation among relevant specialties (departments of neurosurgery, endocrinology, radiation oncology, radiology, etc.) is valuable in treating patients with CD; and 2) bilateral inferior petrosal sinus sampling (BIPSS) might help to lateralize the tumor in MRI-negative/equivocal microadenomas.

\section{Methods \\ Patients}

We performed a retrospective study of endoscopic endonasal surgeries of ACTH-secreting pituitary adenomas conducted between May 2014 and April 2018 at Shanghai Huashan Hospital. The study comprised 124 consecutive patients (128 procedures). There were 21 men and 103 women, with a mean patient age of $38.8 \pm 12.3$ years. All patients were diagnosed and treated strictly according to the clinical guidelines of CD in our center (Supplemental Fig. 1). The patients' medical records and imaging studies were reviewed after obtaining approval from the Huashan IRB.

\section{Endocrine Tests}

All patients went through complete assessment in the department of endocrinology before surgery. The diagnosis of CS was based on cushingoid symptoms, an elevated 24-hour urinary free cortisol (UFC) level above the upper limit of the reference range, and a lack of serum cortisol suppression after $1 \mathrm{mg}$ dexamethasone in the absence of other exogenous glucocorticoid use. ${ }^{21,23}$ Surgical exploration was suggested by normal or elevated preoperative plasma ACTH concentrations, a suppressed cortisol level by high-dose dexamethasone suppression test (HDDST), imaging evidence of a pituitary tumor, or a positive BIPSS. CD was confirmed by at least one of the following: 1) immunopathology confirming the diagnosis; 2) clinical and endocrine remission after pituitary surgery; or 3) preoperative BIPSS predicting a pituitary source. ${ }^{21,23}$

\section{Radiological Assessment}

All cases underwent conventional gadolinium-enhanced MRI (cMRI) of the pituitary region before the surgery, and 3D sampling perfection with application-optimized contrasts using different flip angle evolutions (3DSPACE) MRI scanning (spMRI) was further performed in cases in which no tumor or only a suspicious (equivocal) lesion was indicated on the conventional sequences (Fig. 1). ${ }^{36}$ All images were inspected by senior radiologists and neurosurgeons together.

\section{BIPSS}

The indications for BIPSS with desmopressin stimulation included: 1) BIPSS was only considered for patients with diagnosed CS; 2) no tumor was found on conventional MRI; or 3) tumor was found on MRI, but clinical and biochemical examination suggested a possibility of heterotopic ACTH-secreting syndrome (cortisol level not suppressed by HDDST, or ACTH > $200 \mathrm{pg} / \mathrm{ml})$. CD was diagnosed if
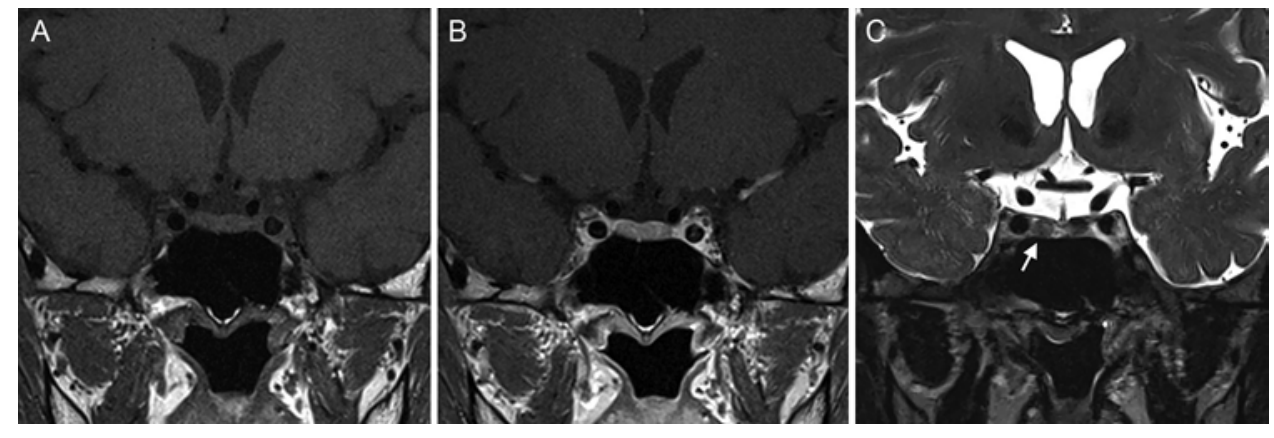

FIG. 1. Coronal MR images of an ACTH-secreting pituitary adenoma. A: T1-weighted image, no tumor detected. B: Contrastenhanced image, no tumor detected. C: 3D-SPACE image, showing the location of the tumor (arrow). 


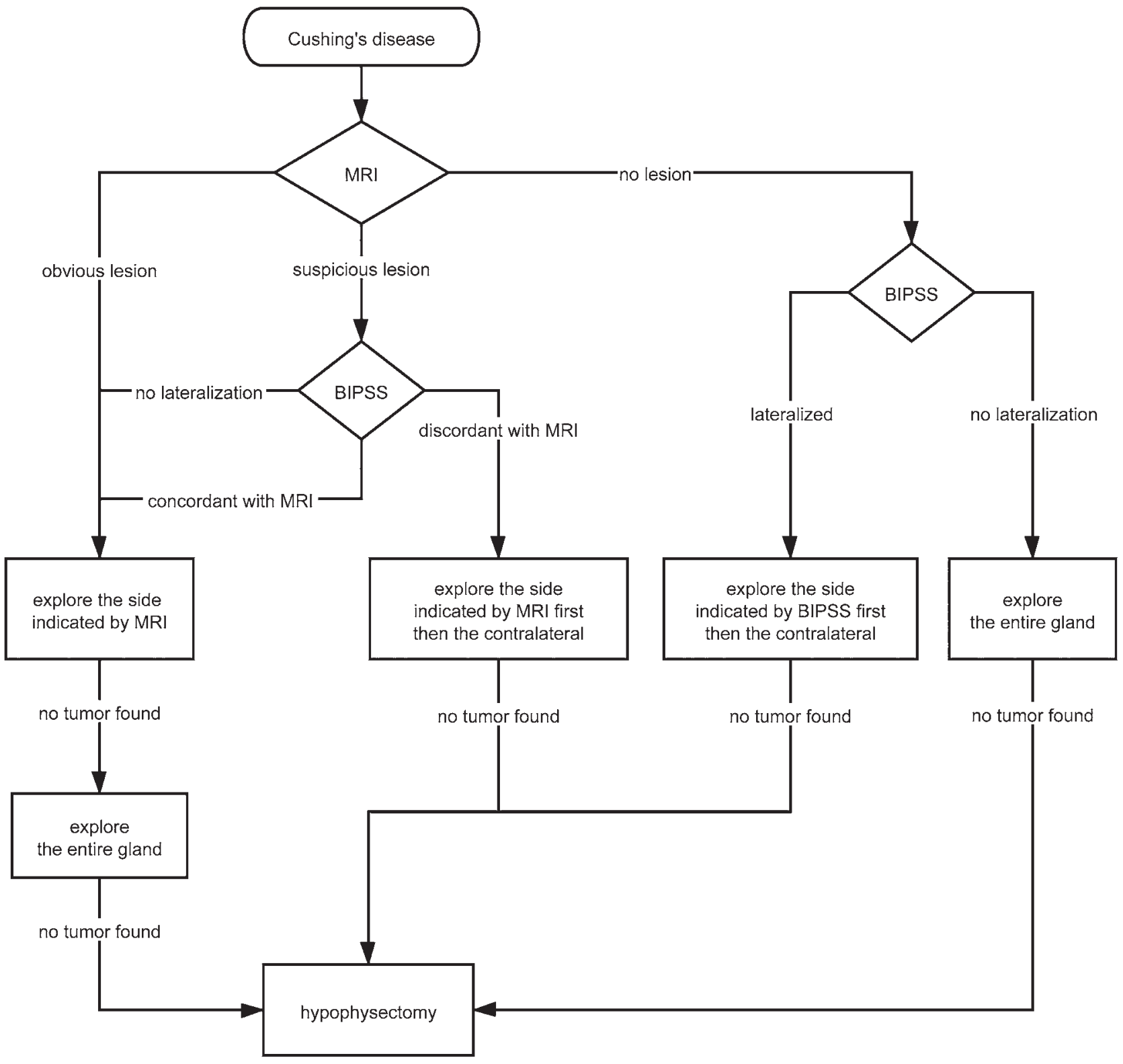

FIG. 2. The surgical strategies for $C D$ based on preoperative lateralization results.

central ACTH levels were two times greater than the peripheral ACTH level at baseline, or three times greater after desmopressin stimulation. Tumor lateralization was indicated if the intersinus ratio was greater than 1.4. ${ }^{18,19,35}$

\section{Surgical Procedure}

All operations were performed using the 4-hand binostril endoscopic endonasal approach. The pituitary gland was explored according to the preoperative pituitary imaging and BIPSS. The extracapsular dissection technique was used in all cases with clear pseudocapsule; otherwise, a thin piece of pituitary tissue surrounding the tumor was resected along with the lesion.

The side of the pituitary gland with a suspicious tumor indicated by the MRI was searched first, followed by the side suggested by the BIPSS (Fig. 2). The contralateral side should be routinely explored to exclude the possibility of multiple lesions, whether the tumor was found in the ipsilateral side or not. In cases with both negative imaging and negative BIPSS lateralization, the entire pituitary gland was carefully explored: several vertical incisions were made deep through the anterior lobe until reaching the neurohypophysis, and laterally until reaching the internal wall of the cavernous sinus on both sides (Fig. 3) ${ }^{9,24}$ If no lesions are found after the exploration, hypophysectomy should be considered, yet none were performed in this study.

\section{Follow-Up and Adjuvant Therapy}

Patients were followed up immediately and at 3 months, 

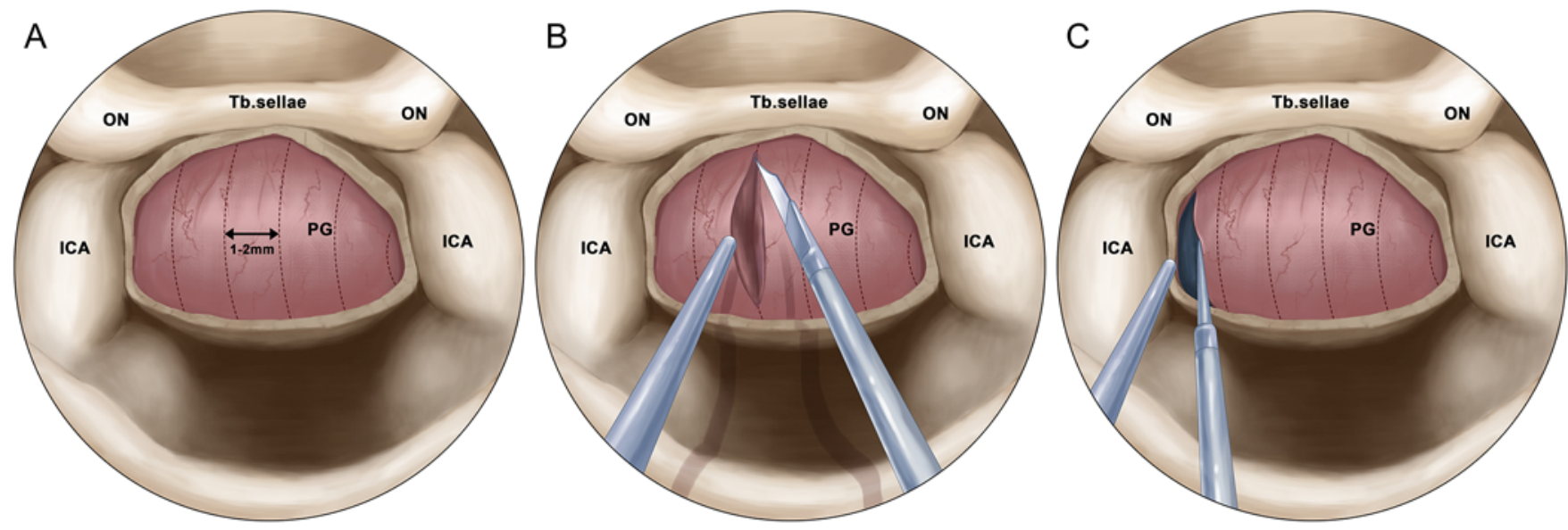

FIG. 3. Illustration of the surgical technique in cases with both negative imaging and BIPSS lateralization. The entire pituitary gland was explored thoroughly by making several vertical incisions (slice thickness 1-2 $\mathrm{mm}$; $\mathbf{A}$ ), deep enough until the posterior lobe was exposed (B), and laterally until the internal wall of the cavernous sinus on both sides was exposed $(\mathbf{C})$. ICA = internal carotid artery; $\mathrm{ON}=$ optic nerve; $\mathrm{PG}=$ pituitary gland; Tb.sellae = tuberculum sellae. Copyright Keyi Zhang. Published with permission.

6 months, and annually after surgery by undergoing complete reassessment in the department of endocrinology at our center. No glucocorticoids were used before or during the operation. The morning serum cortisol level was measured daily before discharge after the surgery. Patients were considered to be in early remission if the nadir serum cortisol level was less than $5 \mu \mathrm{g} / \mathrm{dl}$, and glucocorticoid replacements were given. At 3-months' follow-up, surgical remission was defined as normalized serum cortisol and UFC levels, which could be suppressed by the low-dose dexamethasone suppression test (LDDST). Remission cases who did not achieve early remission were considered to be in delayed remission. Those who failed to meet the criteria above at the 3-month follow-up were judged to be in persistent disease. Other endocrine examinations were based on the 2016 American Endocrine Society guidelines for the diagnosis and treatment of hypophysis. ${ }^{13}$

The adjuvant therapy of patients in persistent disease was discussed by our MDT. For patients with detectable residual tumors on imaging, a second surgery or stereotactic radiotherapy (SRT; Gamma Knife) were suggested. For patients without detectable residual tumors, medical treatment with steroidogenesis inhibitors was recommended. Patients were considered to have a recurrence after postoperative remission if endocrine examination showed hypercortisolism (elevated serum cortisol level not suppressed by LDDST, and elevated UFC) during follow-up.

\section{Statistical Analysis}

Data are presented as means \pm standard deviations for continuous variables normally or not normally distributed, and as frequencies for categorical variables. Normality was tested using the Kolmogorov-Smirnov test. Means were compared using the unpaired t-test when data distribution was normal, or by the Wilcoxon rank-sum (MannWhitney) test when variables were not normally distributed. For categorical variables, differences were analyzed by the chi-square test or Fisher's exact test as appropriate. Odds ratios (ORs) and 95\% CIs were calculated us- ing univariate logistic regression. Clinical covariates that predicted remission with a univariate $\mathrm{p}$ value $<0.15$ were subjected to multivariable logistic regression analysis. Statistical analysis was performed using SPSS statistical software (version 23.0, IBM Corp.). A 2-tailed $\mathrm{p}$ value $<0.05$ was considered significant.

\section{Results}

\section{Patient and Tumor Characteristics}

The characteristics of the study population are summarized in Table 1. Four of the 124 patients $(1$ man and 3 women) underwent reoperation due to persistent disease after the initial surgery. The mean interval between the two operations was 7 months $(3,5,8$, and 12 months, respectively). Therefore, 128 surgeries in total were performed, among which 99 (77.3\%) were initial operations without a history of previous pituitary surgery, and 29 (22.7\%) were reoperations for persistent or recurrent cases.

Before surgery, all cases showed cushingoid symptoms. Central obesity, hypertension, menstrual disorder, acne, purple striae, hirsutism, impaired glucose metabolism, edema, sanguineous appearance, ecchymosis, and increased thirst and urination were found in $92.2 \%, 57.8 \%$, $53.6 \%, 34.4 \%, 26.6 \%, 26.6 \%, 24.2 \%, 24.2 \%, 21.1 \%, 10.9 \%$, and $6.3 \%$ of cases, respectively.

Sixty-two adenomas $(62 / 128,48.4 \%)$ could be identified using cMRI, including $20(15.6 \%)$ macroadenomas and $42(32.8 \%)$ cMRI-visible microadenomas. Another 66 (51.6\%) cases with MRI-equivocal tumors were assessed by BIPSS with desmopressin stimulation, including 44 cases with spMRI-visible lesions, and 22 cases with negative imaging.

Sixty-five $(98.5 \%)$ of the 66 BIPSS cases indicated hypercortisolism from a pituitary source. The other case of BIPSS-negative CD was confirmed by pathology and subsequent surgical remission. Thus, the diagnostic sensitivity of BIPSS was $98.5 \%$ (65/66). Furthermore, 52 $(80 \%)$ of the 65 BIPSS-positive cases showed lateraliza- 
TABLE 1. Baseline characteristics of the entire cohort $(n=128)$

\begin{tabular}{|c|c|}
\hline Characteristic & Value (\%) \\
\hline Mean age $\pm S D$, yrs & $38.8 \pm 12.3$ \\
\hline Males & $22(17.2)$ \\
\hline \multicolumn{2}{|l|}{ Clinical features } \\
\hline Central obesity & $118(92.2)$ \\
\hline Hypertension & $74(57.8)$ \\
\hline Oligomenorrhoea or amenorrhea & $45(53.6)^{*}$ \\
\hline Acne & $44(34.4)$ \\
\hline Purple striae & $34(26.6)$ \\
\hline Hirsutism & $34(26.6)$ \\
\hline Impaired glucose tolerance or diabetes & $31(24.2)$ \\
\hline Edema & $31(24.2)$ \\
\hline Sanguineous appearance & $27(21.1)$ \\
\hline Ecchymosis & $14(10.9)$ \\
\hline Increased thirst \& urination & $8(6.3)$ \\
\hline \multicolumn{2}{|l|}{ History of previous treatment } \\
\hline Pituitary surgery & $22(17.2)$ \\
\hline Radiotherapy & $3(2.3)$ \\
\hline Adrenal surgery & $3(2.3)$ \\
\hline Pituitary surgery + radiotherapy & $2(1.6)$ \\
\hline Pituitary surgery + radiotherapy + adrenal surgery & $2(1.6)$ \\
\hline Pituitary surgery + medical therapy & $3(2.3)$ \\
\hline \multicolumn{2}{|l|}{ History of previous pituitary surgery } \\
\hline No & $99(77.3)$ \\
\hline Yes & $29(22.7)$ \\
\hline \multicolumn{2}{|l|}{ Size of tumor } \\
\hline MRI-negative & $22(17.2)$ \\
\hline MRI-visible microadenoma & $86(67.2)$ \\
\hline Macroadenoma & $20(15.6)$ \\
\hline \multicolumn{2}{|l|}{ Invasiveness of tumor } \\
\hline Noninvasive & $115(89.8)$ \\
\hline Cavernous sinus invasion & $10(7.8)$ \\
\hline Pituitary stalk invasion & $3(2.3)$ \\
\hline \multicolumn{2}{|l|}{ Pathology } \\
\hline ACTH $(+)$ & $126(98.4)$ \\
\hline ACTH (-), T-pit (+)† & $2(1.6)$ \\
\hline \multicolumn{2}{|l|}{ Ki-67 index } \\
\hline $\mathrm{Ki}-67<3 \%$ & $104(81.3)$ \\
\hline $3 \% \leq \mathrm{Ki}-67<10 \%$ & $22(17.2)$ \\
\hline $\mathrm{Ki}-67 \geq 10 \%$ & $2(1.6)$ \\
\hline
\end{tabular}

* Only women of childbearing age (ages $15-49$ years) were included $(n=84)$. $\dagger$ T-pit is a T-box transcription factor restricted to pituitary proopiomelanocortin-expressing cells in humans.

tion. Concordance and discordance with intraoperative lateralization were found in $44(84.6 \%)$ and $4(7.7 \%)$ cases, respectively. Multiple lesions were found in 2 cases (3.8\%), and no tumor was found after complete exploration in the other 2 cases (3.8\%). Thus, the concordance of BIPSS in the preoperative lateralization of CD was $84.6 \%$ (44/52; Supplemental Table 1).

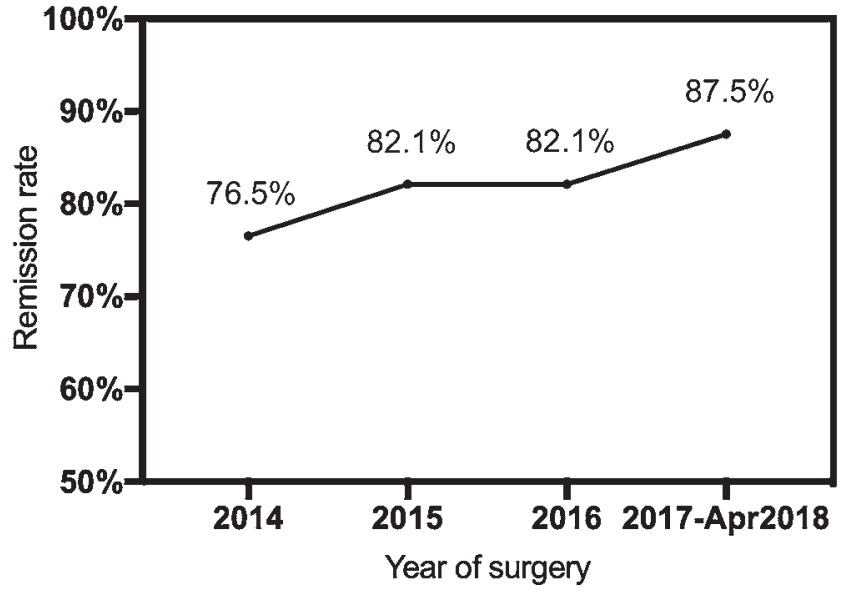

FIG. 4. Learning curve of endoscopic endonasal surgery for $C D$ of a single surgical team in our center from 2014 to 2018: the remission rate of each year appears to be ascending, with a lowest rate of $76.5 \%$ (in $2014,13 / 17$ ) and a highest rate of $87.5 \%$ (2017 to April 2018, 35/40).

\section{Surgical Outcomes and Predictors of Remission}

Early remission was achieved in $102(79.7 \%)$ of the 128 cases after surgery. One hundred thirteen patients (88.3\%) underwent follow-up for at least 6 months after surgery. The average follow-up duration was $20.3 \pm 12.2$ months (range 6-51 months). In these cases, postoperative remission was achieved in 94 cases $(83.2 \%)$. Among them, 87 cases $(77.0 \%)$ were in early remission (postoperative nadir serum cortisol level $<5 \mu \mathrm{g} / \mathrm{dl}$ ), and 7 cases $(6.2 \%)$ were in delayed remission (serum cortisol suppressed by LDDST at 3-months' follow-up, as well as early remission not achieved). Two cases met the criteria of early remission but were proven to be in persistent disease during followup. Seventeen cases were neither in early nor delayed remission. The postoperative remission rates of macroadenomas, MRI-visible microadenomas, and MRI-negative tumors were $66.7 \%, 89.3 \%$, and $75 \%$, respectively $(\mathrm{p}=$ 0.039 ). The postoperative remission rates of noninvasive and invasive tumors were $86.3 \%$ and $54.5 \%$, respectively $(p=0.025)$. The postoperative remission rates of patients with and without a history of previous surgery were $67.9 \%$ and $88.2 \%$, respectively $(\mathrm{p}=0.027)$. The postoperative remission rates in our center showed a trend of improvement in recent years (Fig. 4). The remission rate was higher in 2017-2018 than in 2014, although it was not a statistically significant difference $(87.5 \%$ vs $76.5 \%, \mathrm{p}=0.517)$, emphasizing the importance of the surgeon's experience.

We divided patients into remission $(\mathrm{n}=94)$ and nonremission $(n=19)$ groups (Table 2). Patients in the remission group were more likely to have no history of previous surgery $(79.8 \%$ vs $52.6 \%, \mathrm{p}=0.027)$, had more MRIvisible microadenomas ( $71.3 \%$ vs $42.1 \%, \mathrm{p}=0.039)$, and had fewer invasive tumors based on surgical observation $(6.4 \%$ vs $26.3 \%, p=0.025)$ than those in the nonremission group. There were no significant differences in age, sex distribution, and preoperative lateralization between the two groups $(\mathrm{p}>0.05)$. Following univariate analysis (Supplemental Table 2), a history of previous pituitary surgery, MRI-visible microadenoma, and tumor invasion 
TABLE 2. Comparison of clinical features between remission and nonremission groups $(n=113)$

\begin{tabular}{|c|c|c|c|}
\hline Feature & Remission, $\mathrm{n}=94$ & Nonremission, $n=19$ & $p$ Value \\
\hline Mean age $\pm S D$, yrs & $38.5 \pm 12.2$ & $37.5 \pm 12.7$ & 0.749 \\
\hline Females, $\mathrm{n}(\%)$ & $80(85.1)$ & $17(89.5)$ & 0.891 \\
\hline \multicolumn{4}{|c|}{ History of previous pituitary surgery, $n(\%)$} \\
\hline No & $75(79.8)$ & $10(52.6)$ & 0.027 \\
\hline Yes & $19(20.2)$ & $9(47.4)$ & \\
\hline \multicolumn{4}{|l|}{ Size of tumor, n (\%) } \\
\hline MRI-negative & $15(16.0)$ & $5(26.3)$ & 0.039 \\
\hline MRI-visible microadenoma & $67(71.3)$ & $8(42.1)$ & \\
\hline Macroadenoma & $12(12.8)$ & $6(31.6)$ & \\
\hline \multicolumn{4}{|l|}{ Tumor invasiveness, $\mathrm{n}(\%)$} \\
\hline Noninvasive & $88(93.6)$ & $14(73.7)$ & 0.025 \\
\hline Invasive* & $6(6.4)$ & $5(26.3)$ & \\
\hline \multicolumn{4}{|l|}{ Preop lateralization, $\mathrm{n}(\%)$} \\
\hline Lateralized & $82(87.2)$ & $16(84.2)$ & 1.000 \\
\hline Nonlateralized & $12(12.8)$ & $3(15.8)$ & \\
\hline
\end{tabular}

were included in the multivariate analysis (Table 3). A history of previous pituitary surgery (OR $0.300,95 \%$ CI 0.100-0.903; $\mathrm{p}=0.032$ ) and MRI-visible microadenoma (OR 3.048, 95\% CI 1.030-9.019; $\mathrm{p}=0.044$ ) were independent factors influencing surgical remission.

\section{Long-Term Follow-Up, Adjuvant Therapy, and Recurrence}

The details of adjuvant therapies are summarized in Table 4. Fifteen (78.9\%) of the 19 cases in persistent disease after surgery were followed up by our MDT. Three, 3,5 , and 4 patients underwent reoperation, SRT, medical therapy, or a combination of two treatment modalities, respectively. Remission was achieved in 10 patients $(66.7 \%)$ after adjunctive therapies. Five patients were still in persistent disease, and one of them died due to intracerebral hemorrhage 14 months after the primary operation. Four patients were followed up by their local hospitals. No adjuvant therapy was adopted for these patients due to the lack of MDT collaboration. All of them were still in persistent disease, and one of them died due to cardiovascular events a year after the primary operation. Therefore, the remission rate of MDT follow-up was higher than that of nonMDT follow-up $(66.7 \%$ vs $0 \%, \mathrm{p}=0.033)$. Three $(3.2 \%)$ of the 94 patients in remission after surgery experienced a

TABLE 3. Multivariate analysis results for predictors of postoperative remission $(n=113)$

\begin{tabular}{lcc}
\hline \multicolumn{1}{c}{ Feature } & OR $(95 \% \mathrm{Cl})$ & $\mathrm{p} \mathrm{Value}$ \\
\hline History of previous pituitary surgery & $0.300(0.100-0.903)$ & $\mathbf{0 . 0 3 2}$ \\
\hline MRI-visible microadenoma & $3.048(1.030-9.019)$ & $\mathbf{0 . 0 4 4}$ \\
\hline Tumor invasion & $0.310(0.075-1.280)$ & 0.105 \\
\hline
\end{tabular}

Boldface type indicates statistical significance $(p<0.05)$. recurrence during follow-up. The mean time to recurrence in these 3 patients was 18 months (10, 19, and 24 months). All the recurrent tumors were found in the cavernous sinus, and adjuvant SRT was adopted. Two of the patients achieved remission afterward, and the other was lost to follow-up. Thus, the overall remission rate until the last investigation was $91.2 \%(103 / 113)$.

\section{Complications}

The mortality rate of this cohort was $1.8 \%$ (2/113). The cause of the two deaths was cardio-cerebrovascular events related to $\mathrm{CD}$. Eleven patients $(8.6 \%, 11 / 128)$ had intraoperative CSF leakages, which were repaired with collagen sponge or autologous fat. One patient $(0.8 \%, 1 / 128)$ experienced mental disorders of anxiety and panic attacks and recovered after psychiatric therapy. One patient $(0.8 \%$, 1/128) experienced acute pulmonary edema and gradually improved after noninvasive positive-pressure ventilation therapy. Fifteen patients $(11.7 \%, 15 / 128)$ had transient diabetes insipidus. Eight patients $(6.3 \%, 8 / 128)$ had the syndrome of inappropriate antidiuretic hormone, including 3 patients $(2.3 \%, 3 / 128)$ with severe hyponatremia below $120 \mathrm{mmol} / \mathrm{L}$. Hypoadrenalism, hypothyroidism, and panhypopituitarism (at least two axes) developed in $20.4 \%$ (23/113), $4.4 \%$ (5/113), and $8.0 \%$ (9/113) of the patients, respectively, and were treated with long-term hormonal replacement. No patient reported deterioration in visual acuity or worsening in their visual field deficit. No incidents of intraoperative carotid injury, ophthalmoplegia, postoperative CSF leakage, meningitis, or permanent diabetes insipidus occurred.

\section{Discussion}

The treatment goals of CD include the following: 1) 
TABLE 4. Adjuvant therapies for patients with persistent disease $(n=19)$

\begin{tabular}{|c|c|c|c|c|c|}
\hline Pt No. & Age (yrs), Sex & FU (mos) & MDT Management & Adjunct Therapy & Outcome \\
\hline 1 & $26, F$ & 51 & Yes & SRT + medical therapy* & Remission \\
\hline 2 & $33, F$ & 47 & No & None & Nonremission \\
\hline 3 & $24, \mathrm{~F}$ & 46 & Yes & SRT & Remission \\
\hline 4 & $48, \mathrm{~F}$ & 47 & No & None & Nonremission \\
\hline 5 & $41, \mathrm{~F}$ & 13 & Yes & SRT & Nonremission \\
\hline 6 & $35, \mathrm{~F}$ & 35 & Yes & Medical therapy & Remission \\
\hline 7 & $61, M$ & 14 & Yes & Adrenalectomy + reoperation $†$ & Dead \\
\hline 8 & $24, \mathrm{~F}$ & 32 & Yes & Medical therapy & Nonremission \\
\hline 9 & $37, \mathrm{~F}$ & 19 & Yes & Reoperation + SRT & Remission \\
\hline 10 & $35, \mathrm{~F}$ & 30 & Yes & Reoperation & Remission \\
\hline 11 & $54, \mathrm{~F}$ & 12 & No & None & Dead \\
\hline 12 & $25, F$ & 25 & Yes & SRT & Remission \\
\hline 13 & $58, \mathrm{~F}$ & 22 & Yes & Reoperation + SRT & Nonremission \\
\hline 14 & $19, \mathrm{~F}$ & 20 & Yes & Medical therapy & Nonremission \\
\hline 15 & $45, \mathrm{~F}$ & 17 & No & None & Nonremission \\
\hline 16 & $47, \mathrm{~F}$ & 17 & Yes & Reoperation & Remission \\
\hline 17 & $46, M$ & 13 & Yes & Medical therapy & Remission \\
\hline 18 & $32, \mathrm{~F}$ & 12 & Yes & Reoperation & Remission \\
\hline 19 & $22, \mathrm{~F}$ & 10 & Yes & Medical therapy & Remission \\
\hline \multicolumn{6}{|c|}{$\begin{array}{l}\text { FU = follow-up; Pt = patient. } \\
\text { * Medical therapy = ketoconazole. } \\
\dagger \text { The adjuvant adrenalectomy and pituitary reoperation of patient } 7 \text { were performed in the local hospital. Remission } \\
\text { was not achieved. The patient died of cerebrovascular events } 14 \text { months after the primary operation. } \\
\text { † The adjuvant pituitary reoperation of patient } 13 \text { was performed in the local hospital. Remission was not achieved until } \\
\text { our MDT suggested SRT. }\end{array}$} \\
\hline
\end{tabular}

normalization of the cortisol levels, 2) an improvement in clinical symptoms, 3) prevention or control of the comorbidities, 4) long-term disease control without recurrence, and 5) elimination of tumor compression on the pituitary gland and other adjacent structures while saving normal pituitary functions. ${ }^{3,4,16,26,32}$ A complete treatment for $\mathrm{CD}$ needs multidisciplinary cooperation. TSS is currently the first-line treatment for $\mathrm{CD}$, including two main approaches: the microscopic transsphenoidal and the endoscopic endonasal approaches. The latter provides a more-detailed view of a close-up observation even in MRI-negative/ equivocal microadenomas. The endoscopic approach also has an improved panoramic visualization that can help to identify important structures and to dissect the tumors invading laterally to the cavernous sinus or superiorly to the suprasellar regions. ${ }^{14,27,30}$

\section{Remission Rate and Recurrence Rate}

In the past 2 decades, the remission rates of $C D$ and MRI-negative CD have been approximately $77.5 \%-84.3 \%$ and $42.9 \%-71 \%$, respectively, in different medical centers, and the recurrence rate was $9.6 \%-15.9 \%$ depending on different follow-up periods. ${ }^{1,6,9,11,22,26,31,37}$ The remission rates of $\mathrm{CD}$ and MRI-negative $\mathrm{CD}$ after surgery alone were $83.2 \%$ and $75 \%$, respectively, in our cohort. The postoperative remission rates in our center showed a trend of improvement in recent years, although not statistically significant $(87.5 \%$ in $2017-2018$ vs $76.5 \%$ in 2014 , $p=0.517)$, emphasizing the importance of the surgeon's experience. The relatively high remission rates in our cohort were probably related to the following experiences we would like to share: 1) the diagnosis of CD should be confirmed before surgical exploration, which rules out the chance of CS from nonpituitary sources; 2) a thorough exploration of the entire pituitary gland should be performed with patience, especially in regions near the medial wall of the cavernous sinus and below the diaphragma sellae; and 3) extracapsular dissection or $101 \%$ resection should be adopted to minimize the chance of tumor remnants. The recurrence rate was $3.2 \%$ in our cohort. The low incidence of recurrence might be related to the relatively short follow-up period in this study (20.3 \pm 12.2 months).

Current systematic reviews and meta-analyses indicated that macroadenomas, invasive adenomas, MRI-negative tumors, and cases performed by surgeons with limited experience were less likely to achieve remission. ${ }^{1,4,15,26,28,32}$ The results of our study were generally concordant with the literature. MRI-visible microadenomas, noninvasive adenomas, and cases without a history of previous pituitary surgery were found to be more likely to achieve remission by univariate analysis. After multivariate analysis, the independent factors influencing surgical remission in CD were a history of previous pituitary surgery (OR 0.300 , 95\% CI 0.100-0.903; $\mathrm{p}=0.032$ ) and MRI-visible microadenoma (OR 3.048, 95\% CI 1.030-9.019; $\mathrm{p}=0.044$ ). It is not surprising that MRI-visible microadenoma is an inde- 
pendent predictor because it is relatively easy to resect a microadenoma when you know where it is. Future imaging studies should be carried out to help localize the tumor before surgery. For the other independent predictor, we speculate that it might be related to the following factors: $1)$ the relatively high proportion of reoperations $(22.7 \%)$ in our cohort magnifies its influence; 2) the scar generated by previous pituitary surgery makes a thorough exploration of the entire pituitary gland more challenging; and 3) the prior surgery may split the tumor into several pieces, adding difficulty to the total resection during reoperation.

\section{The Concordance of BIPSS Lateralization With Intraoperative Observations}

Accurate preoperative localization provides reliable guidance for surgical procedures, which might help to improve the remission rate, minimize the damage to the normal pituitary gland, and shorten the operative duration. However, accurate localization is still problematic, although new MRI techniques such as 3D-SPACE imaging have improved the detectable rate of MRI-negative/ equivocal adenomas. ${ }^{17,29,33,34,36}$

For MRI-negative/equivocal cases, BIPSS with desmopressin stimulation tests were necessary for both diagnosis and tumor lateralization. The BIPSS test is currently recognized as a gold standard in the diagnosis of CD, with a sensitivity of approximately $92.1 \%-98.9 \% .^{10,12,25,31}$ In our study, the diagnostic sensitivity of BIPSS was $98.5 \%$. The BIPSS with the desmopressin stimulation test might have a role in tumor lateralization, which is important guidance for surgical exploration. The reported accuracy of tumor lateralization by BIPSS was $54.0 \%-78.7 \%$ in the literature..$^{10,12,35}$ The causes of the discordance between BIPSS and intraoperative observations include an anatomical variation of the inferior petrosal sinus and catheter positioning. ${ }^{12,18,19,35}$ The accuracy of tumor lateralization by BIPSS was $84.6 \%$ in our study, which suggests that BIPSS might help to lateralize the tumor in MRI-negative/equivocal cases. Due to the relatively small sample size of BIPSS in the present study, it still needs to be verified in largersample prospective trials.

\section{Multidisciplinary Cooperation}

The diagnosis and management of CD requires multidisciplinary collaboration, especially for patients with persistent disease or recurrence after one or multiple surgical procedures. ${ }^{2,20}$ Our MDT for pituitary tumors was first established in 2013, and the cooperation between our specialists from different departments has been increasingly intimate ever since. Furthermore, we created a "Gold Pituitary" joint ward in our medical center in December 2018 to provide the best care for patients with pituitary tumors and meet the criteria of Pituitary Tumors Centers of Excellence. ${ }^{5}$ Neurosurgeons and endocrinologists work seamlessly in the same ward. We make joint ward rounds together and arrange a multidisciplinary discussion on complex cases once a week. Up to 16 experts from other specialties, including radiation oncologists, radiologists, ophthalmologists, otorhinolaryngologists, gynecologists, andrologists, pathologists, infectious disease physicians, and laboratory scientists have signed up as members of our
MDT and will join the discussion when necessary. In our cohort, the remission rate of postoperative MDT management in patients with persistent disease was higher than non-MDT management $(66.7 \%$ vs $0 \%, \mathrm{p}=0.033)$, which preliminarily reflected the value of multidisciplinary cooperation.

The strength of the present study is the relatively large size of the purely endoscopic endonasal surgical cohort within a consecutive 4-year period, which shows the learning curve nicely with the improvement of surgical results and helps to reveal factors influencing remission after surgery alone. The limitations of this study included its retrospective nature and the fact that we had a relatively short follow-up period to estimate the long-term recurrence rate. We speculate that the short period of follow-up might be related to the fact that approximately $80 \%$ of our patients come from other cities outside of Shanghai. It is difficult for everyone to visit us after 1-2 years of remission after surgery.

\section{Conclusions}

Endoscopic endonasal surgery is a safe and efficient procedure in the treatment of CD. MRI-visible microadenoma and primary surgery are independent predictors of surgical remission. The MDT management strategy helps to achieve a better overall outcome. BIPSS may help to lateralize the tumor in MRI-negative/equivocal microadenomas.

\section{Acknowledgments}

This work was supported by the Science and Technology Commission of Shanghai Municipality (grant no. 17441901400 received by Yongfei Wang, $\mathrm{MD}, \mathrm{PhD}$ ) and by the National Natural Science Foundation of China (grant no. 81602191 received by Ming Shen, MD, PhD).

\section{References}

1. Alexandraki KI, Kaltsas GA, Isidori AM, Storr HL, Afshar F, Sabin I, et al: Long-term remission and recurrence rates in Cushing's disease: predictive factors in a single-centre study. Eur J Endocrinol 168:639-648, 2013

2. Bertagna X, Guignat L: Approach to the Cushing's disease patient with persistent/recurrent hypercortisolism after pituitary surgery. J Clin Endocrinol Metab 98:1307-1318, 2013

3. Bertagna X, Guignat L, Groussin L, Bertherat J: Cushing's disease. Best Pract Res Clin Endocrinol Metab 23:607623, 2009

4. Biller BM, Grossman AB, Stewart PM, Melmed S, Bertagna $\mathrm{X}$, Bertherat J, et al: Treatment of adrenocorticotropin-dependent Cushing's syndrome: a consensus statement. J Clin Endocrinol Metab 93:2454-2462, 2008

5. Casanueva FF, Barkan AL, Buchfelder M, Klibanski A, Laws ER, Loeffler JS, et al: Criteria for the definition of Pituitary Tumor Centers of Excellence (PTCOE): a Pituitary Society statement. Pituitary 20:489-498, 2017

6. Ciric I, Zhao JC, Du H, Findling JW, Molitch ME, Weiss RE, et al: Transsphenoidal surgery for Cushing disease: experience with 136 patients. Neurosurgery 70:70-81, 2012

7. Clayton RN, Jones PW, Reulen RC, Stewart PM, HassanSmith ZK, Ntali G, et al: Mortality in patients with Cushing's disease more than 10 years after remission: a multicentre, multinational, retrospective cohort study. Lancet Diabetes Endocrinol 4:569-576, 2016 
8. Clayton RN, Raskauskiene D, Reulen RC, Jones PW: Mortality and morbidity in Cushing's disease over 50 years in Stoke-on-Trent, UK: audit and meta-analysis of literature. J Clin Endocrinol Metab 96:632-642, 2011

9. Dallapiazza RF, Oldfield EH, Jane JA Jr: Surgical management of Cushing's disease. Pituitary 18:211-216, 2015

10. Deipolyi A, Bailin A, Hirsch JA, Walker TG, Oklu R: Bilateral inferior petrosal sinus sampling: experience in 327 patients. J Neurointerv Surg 9:196-199, 2017

11. Feng M, Liu Z, Liu X, Bao X, Yao Y, Deng K, et al: Diagnosis and outcomes of 341 patients with Cushing's disease following transsphenoid surgery: a single-center experience. World Neurosurg 109:e75-e80, 2018

12. Feng M, Liu Z, Liu X, Zhang X, Bao X, Yao Y, et al: Tumour lateralization in Cushing's disease by inferior petrosal sinus sampling with desmopressin. Clin Endocrinol (Oxf) 88:251-257, 2018

13. Fleseriu M, Hashim IA, Karavitaki N, Melmed S, Murad MH, Salvatori R, et al: Hormonal replacement in hypopituitarism in adults: an Endocrine Society Clinical Practice Guideline. J Clin Endocrinol Metab 101:3888-3921, 2016

14. Frank G, Pasquini E, Farneti G, Mazzatenta D, Sciarretta V, Grasso V, et al: The endoscopic versus the traditional approach in pituitary surgery. Neuroendocrinology 83:240248, 2006

15. Johnston PC, Kennedy L, Hamrahian AH, Sandouk Z, Bena J, Hatipoglu B, et al: Surgical outcomes in patients with Cushing's disease: the Cleveland clinic experience. Pituitary 20:430-440, 2017

16. Lacroix A, Feelders RA, Stratakis CA, Nieman LK: Cushing's syndrome. Lancet 386:913-927, 2015

17. Lee HB, Kim ST, Kim HJ, Kim KH, Jeon P, Byun HS, et al: Usefulness of the dynamic gadolinium-enhanced magnetic resonance imaging with simultaneous acquisition of coronal and sagittal planes for detection of pituitary microadenomas. Eur Radiol 22:514-518, 2012

18. Lefournier V, Martinie M, Vasdev A, Bessou P, Passagia JG, Labat-Moleur F, et al: Accuracy of bilateral inferior petrosal or cavernous sinuses sampling in predicting the lateralization of Cushing's disease pituitary microadenoma: influence of catheter position and anatomy of venous drainage. J Clin Endocrinol Metab 88:196-203, 2003

19. Liu C, Lo JC, Dowd CF, Wilson CB, Kunwar S, Aron DC, et al: Cavernous and inferior petrosal sinus sampling in the evaluation of ACTH-dependent Cushing's syndrome. Clin Endocrinol (Oxf) 61:478-486, 2004

20. Liubinas SV, Porto LD, Kaye AH: Management of recurrent Cushing's disease. J Clin Neurosci 18:7-12, 2011

21. Loriaux DL: Diagnosis and differential diagnosis of Cushing's syndrome. N Engl J Med 376:1451-1459, 2017

22. Mehta GU, Lonser RR: Management of hormone-secreting pituitary adenomas. Neuro Oncol 19:762-773, 2017

23. Newell-Price J, Bertagna X, Grossman AB, Nieman LK: Cushing's syndrome. Lancet 367:1605-1617, 2006

24. Oldfield EH, Vortmeyer AO: Development of a histological pseudocapsule and its use as a surgical capsule in the excision of pituitary tumors. J Neurosurg 104:7-19, 2006

25. Pecori Giraldi F, Cavallo LM, Tortora F, Pivonello R, Colao A, Cappabianca P, et al: The role of inferior petrosal sinus sampling in ACTH-dependent Cushing's syndrome: review and joint opinion statement by members of the Italian Society for Endocrinology, Italian Society for Neurosurgery, and Italian Society for Neuroradiology. Neurosurg Focus 38(2):E5, 2015

26. Pivonello R, De Leo M, Cozzolino A, Colao A: The treatment of Cushing's disease. Endocr Rev 36:385-486, 2015

27. Powell M: Microscope and endoscopic pituitary surgery. Acta Neurochir (Wien) 151:723-728, 2009
28. Roelfsema F, Biermasz NR, Pereira AM: Clinical factors involved in the recurrence of pituitary adenomas after surgical remission: a structured review and meta-analysis. Pituitary 15:71-83, 2012

29. Rossi Espagnet MC, Bangiyev L, Haber M, Block KT, Babb J, Ruggiero V, et al: High-resolution DCE-MRI of the pituitary gland using radial k-space acquisition with compressed sensing reconstruction. AJNR Am J Neuroradiol 36:14441449,2015

30. Swearingen B: Update on pituitary surgery. J Clin Endocrinol Metab 97:1073-1081, 2012

31. Tritos NA, Biller BM: Cushing's disease. Handb Clin Neurol 124:221-234, 2014

32. Tritos NA, Biller BM, Swearingen B: Management of Cushing disease. Nat Rev Endocrinol 7:279-289, 2011

33. Vitale G, Tortora F, Baldelli R, Cocchiara F, Paragliola RM, Sbardella E, et al: Pituitary magnetic resonance imaging in Cushing's disease. Endocrine 55:691-696, 2017

34. Wang J, Wu Y, Yao Z, Yang Z: Assessment of pituitary micro-lesions using 3D sampling perfection with applicationoptimized contrasts using different flip-angle evolutions. Neuroradiology 56:1047-1053, 2014

35. Wind JJ, Lonser RR, Nieman LK, DeVroom HL, Chang R, Oldfield EH: The lateralization accuracy of inferior petrosal sinus sampling in 501 patients with Cushing's disease. J Clin Endocrinol Metab 98:2285-2293, 2013

36. Wu Y, Wang J, Yao Z, Yang Z, Ma Z, Wang Y: Effective performance of contrast enhanced SPACE imaging in clearly depicting the margin of pituitary adenoma. Pituitary 18:480-486, 2015

37. Yamada S, Inoshita N, Fukuhara N, Yamaguchi-Okada M, Nishioka H, Takeshita A, et al: Therapeutic outcomes in patients undergoing surgery after diagnosis of Cushing's disease: a single-center study. Endocr J 62:1115-1125, 2015

\section{Disclosures}

The authors report no conflict of interest concerning the materials or methods used in this study or the findings specified in this paper.

\section{Author Contributions}

Conception and design: Wang, K Zhang. Acquisition of data: K Zhang. Analysis and interpretation of data: K Zhang. Drafting the article: K Zhang. Critically revising the article: Wang, Shen, Qiao, Chen, W He, Ye. Approved the final version of the manuscript on behalf of all authors: Wang. Statistical analysis: K Zhang, Shen, Qiao. Administrative/technical/material support: Shen, Qiao, Chen, W He, Ma, Shou, S Li, Zhao, Pan, Liu, M He, Z Zhang, Y Li, Yao, Ye. Study supervision: all authors.

\section{Supplemental Information}

\section{Online-Only Content}

Supplemental material is available online.

Supplemental Figure and Tables. https://thejns.org/doi/suppl/ 10.3171/2020.3.FOCUS2067.

\section{Correspondence}

Yongfei Wang: Shanghai Huashan Institute of Neurological Surgery, Huashan Hospital, Shanghai Medical School, Fudan University, Shanghai, China.eamns@hotmail.com. 